\section{Fortgeschrittener GIST: Schlechtere Prognose nach Absetzen von Imatinib}

\author{
Wird Imatinib bei Patienten mit fortgeschrittenem GIST abgesetzt, schreitet \\ die Krankheit meist rasch voran. Wie sieht der Krankheitsverlauf nach \\ Wiedereinsetzen der Therapie bei den Patienten im Progress aus?
}

D er Tyrosinkinasehemmer Imatinib hat die Behandlung des fortgeschrittenen gastrointestinalen Stromatumors (GIST) wesentlich verbessert. Nach median 20-24 Monaten Dauerbehandlung kann jedoch eine sekundäre Resistenz auftreten, meist hervorgerufen durch neu aufgetretene Mutationen des KITGens und die damit verbundene Synthese von Imatinib-resistenten Proteinen. In der BFR14-Studie wurde deshalb untersucht, wie sich eine gezielte Unterbrechung der Imatinib-Medikation bei 71 Patienten mit fortgeschrittenem GIST nach 1, 3 oder 5 Jahren auswirkt. Wichtigstes Ergebnis der Studie: meist schreitet die Erkrankung dann rasch voran.

In einer weiteren Analyse dieser randomisierten Phase-III-Studie wurde nun der weitere Krankheitsverlauf nach Wiedereinsetzen der Imatinib-Behandlung untersucht. Zum Analysezeitpunkt hatten 51 der 71 Patienten die Imatinib-Therapie nach diagnostiziertem Krankheitsprogress fortgesetzt. Bei 18 Patienten (35\%) trat der Progress lediglich bei den bekannten Läsionen auf. 33 Patienten (65\%) hatten neue Läsionen, bei 17 dieser Patienten schritt der Tumor auch bei den bekannten Läsionen voran. Nur 8 (42\%) der Patienten mit einer kompletten Response (CR) und 12 (52\%) mit einer partiellen Response (PR) zum Randomisationszeitpunkt erreichten eine neue CR und PR. Patienten, die nach Absetzen von Imatinib rasch progredient wurden, hatten eine schlechtere Prognose. Der Tumorstatus zum Zeitpunkt der Randomisation beeinflusste die Zeit bis zum Progress nach Wiederaufnahme der Medikation.

Fazit: Patienten mit fortgeschrittenem GIST, die nach Absetzen von Imatinib einen Progress erleiden, erreichen nach Wiederaufnahme der Behandlung häufig keine Response wie unter der Ersttherapie. Eine rasche Progression nach Imatinib-Stopp ist mit einem schlechteren progressionsfreien Überleben nach Medikationswiederaufnahme verbunden.

Barbara Kreutzkamp

Patrikidou A et al. Influence of imatinib interruption and rechallenge on the residual disease in patients with advanced GIST: results of the BFR14 prospective French Sarcoma Group randomised, phase III trial. Ann Oncol. 2013;24(4):1087-93.

Kommentar von PD Dr. Reichardt: Eine nach korrekter Indikationsstellung begonnene Imatinib-Therapie sollte bei Patienten mit fortgeschrittenem oder metastasiertem GIST zu keinem Zeitpunkt beendet werden, es sei denn, nicht beherrschbare schwere Nebenwirkungen erfordern diese Maßnahme - was glücklicherweise nur äußerst selten vorkommt. Dies ist die eindeutige Botschaft der französischen Studie BFR14. Fast alle Patienten entwickelten nach Therapiebeendigung ein Rezidiv oder einen Krankheitsprogress, abhängig vom Status der Er-

\section{„Die Imatinib-Therapie sollte möglichst nicht unterbrochen werden."}

krankung vor initialer Einleitung der Therapie. Nach 1-oder 3-jähriger Behandlung trat der Progress median nach ca. sechs Monaten ein, nach 5-jähriger Behandlung dagegen erst nach ca. zwölf Monaten. Dies spricht dafür, dass sich mit längerer Behandlungsdauer das Potenzial für ein Rezidiv oder einen Progress zwar von der Kinetik abschwächt, die grundsätzliche Gefahr aber bestehen bleibt. Daraus ergibt sich eine interessante Analogie zum langfristigen Verlauf unter ununterbrochener Imatinib-Therapie. In der Studie B2222 zeigte sich, dass in den ersten Jahren der Imatinib-Behandlung bei Patienten mit metastasiertem GIST das Progressionsrisiko infolge einer Sekundärresistenz bei ca. $20 \%$ pro Jahr liegt. Nach 6-7 Jahren ununterbrochener Behandlung bei wenigstens stabiler Erkrankung fällt das Risiko einer Progression dann aber auf unter $5 \%$ [von Mehren M et al. J Clin Oncol. 2011;
29(suppl):Abstr. 10016]. Daraus lässt sich folgern, dass mit zunehmender Behandlungsdauer das sekundäre Progressionsrisiko unter fortlaufender Therapie immer geringer wird, bei Absetzen der Therapie aber zumindest auch nach fünf Jahren noch nahezu obligat ein Rezidiv droht. Um die Frage der Therapiedauer auch hinsichtlich weit längerer Behandlungszeiträume beantworten zu können, plant die französische Studiengruppe nach zehn Jahren Behandlung eine erneute Randomisierung.

Hinsichtlich des Gesamtüberlebens ergibt sich in der BFR14-Studie bislang kein Unterschied zwischen den Behandlungsgruppen. Dies spricht für die "Wirksamkeit des Imatinib-Rescues", also des Wiederbeginns der Behandlung bei Progression. Statistisch einschränkend muss aber festgestellt werden, dass die Fallzahlberechnung der Studie nicht auf die Detektion eines Unterschieds im Gesamtüberleben angelegt war und die Antwort auf diese Frage letztendlich offen bleiben muss.

Der zumindest nicht nachgewiesene Überlebensnachteil mag indes den einen oder anderen Behandler oder auch Patienten dazu verleitet haben, bei gutem Behandlungserfolg entgegen der klaren Leitlinienempfehlung die Imatinib-Therapie abzusetzen und bei - nahezu obligater - erneuter Progression die Behandlung erneut zu beginnen und damit Gesamtbehandlungsdauer "einzusparen“. Die jetzt nachgewiesene schlechtere Remissionsqualität bei Wiederaufnahme der Imatinib-Therapie nach Behandlungspause mahnt hierzu eindrucksvoll zur Vorsicht. Auch wenn es schwerlich statistisch nachzuweisen sein wird, bleibt doch die Gefahr bestehen, dass sich eine Therapiepause nachteilig auf den Gesamtbehandlungserfolg auswirkt. Somit stellt die vorgelegte Publikation ein weiteres starkes Argument für eine kontinuierliche Dauertherapie mit Imatinib bei Patienten mit metastasiertem GIST dar. Dies gilt auch für den Fall einer sekundären Resektion von residuellen Metastasen und dadurch erreichter Tumorfreiheit in der Bildgebung.

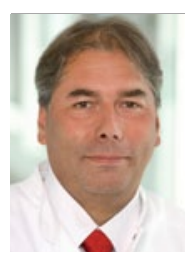

PD Dr. Peter Reichardt HELIOS Klinikum BerlinBuch, Sarkomzentrum Berlin-Brandenburg peter.reichardt@helioskliniken.de 